\title{
THE LSB THEOREM IMPLIES THE KKM LEMMA
}

\author{
GWEN SPENCER AND FRANCIS EDWARD SU
}

Let $S^{d}$ be the unit $d$-sphere, the set of all points of unit Euclidean distance from the origin in $\mathbb{R}^{d+1}$. Any pair of points in $S^{d}$ of the form $x,-x$ is a pair of antipodes in $S^{d}$. Let $\Delta^{d}$ be the $d$-simplex formed by the convex hull of the standard unit vectors in $\mathbb{R}^{d+1}$. Equivalently, $\Delta^{d}=\left\{\left(x_{1}, \ldots, x_{d+1}\right)\right.$ : $\left.\sum_{i} x_{i}=1, x_{i} \geq 0\right\}$. The following are two classical results about closed covers of these topological spaces:

The LSB Theorem (Lusternik-Schnirelman-Borsuk [6] 3]). Suppose that $S^{d}$ is covered by $d+1$ closed sets $A_{1}, \ldots, A_{d+1}$. Then some $A_{i}$ contains a pair of antipodes.

The KKM Lemma (Knaster-Kuratowski-Mazurkiewicz [5]). Suppose that $\Delta^{d}$ is covered by $d+1$ closed sets $C_{1}, C_{2}, \ldots C_{d+1}$ such that for each $x$ in $\Delta^{d}, x$ is in $\cup\left\{C_{i}: x_{i}>0\right\}$. Then all the sets have a common intersection point, i.e., $\cap_{i=1}^{d+1} C_{i}$ is non-empty.

A cover satisfying the condition in the KKM lemma is sometimes called a KKM cover. It can be rephrased in an alternate way: associate labels $1,2, . ., d+1$ to the vertices of $\Delta^{d}$; then demand that vertex $i$ is covered by set $C_{i}$ and that each face of $\Delta^{d}$ is covered by the sets that correspond to the vertices spanning that face.

Both of the above set-covering results are perhaps best known in connection with their equivalent formulations in topology; the LSB theorem is equivalent to the Borsuk-Ulam theorem [3], and the KKM lemma is equivalent to the Brouwer fixed point theorem [5]. Also, the LSB theorem has found spectacular application in proofs of the Kneser conjecture in combinatorics [1, 4]. The KKM lemma has numerous applications in economics, e.g., see [2].

Since the Brouwer fixed point theorem can be obtained as a consequence of the Borsuk-Ulam theorem [7], it is natural to ask if the there is a proof of the KKM lemma using the LSB theorem. The purpose of this note is to provide such a proof.

Theorem. The LSB theorem implies the KKM lemma.

Observe that the LSB theorem holds for a $d$-sphere under any metric on $\mathbb{R}^{d+1}$, since such a sphere and $S^{d}$ are related by an antipode-preserving homeomorphism. In particular, the LSB theorem holds for a $d$-sphere under the $L^{1}$ norm:

$$
\Sigma^{d}:=\left\{\left(x_{1}, \ldots, x_{d+1}\right): \sum_{i}\left|x_{i}\right|=1\right\} .
$$

For $d=2$, this is just the boundary of a regular octahedron, and for general $d, \Sigma^{d}$ is the boundary of the $(d+1)$-crosspolytope. It is the union of $2^{d+1}$ facets which are simplices, one for each orthant of $\mathbb{R}^{d+1}$. See Figure 1

It will be convenient, then, to use the LSB theorem for $\Sigma^{d}$ to prove the KKM lemma, because $\Delta^{d}$ is naturally embedded in $\Sigma^{d}$; namely, $\Delta^{d}$ is the facet of $\Sigma^{d}$ for which $\sum_{i} x_{i}=1$. Call this facet 


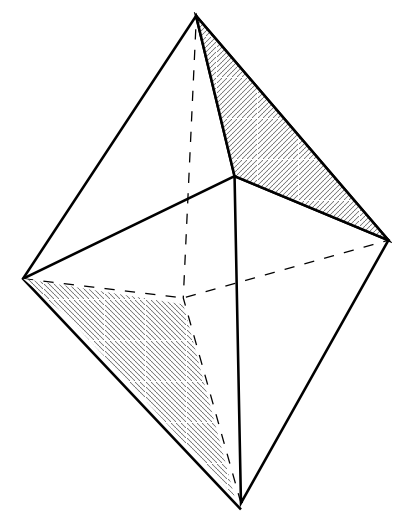

Figure 1 . The 2 -sphere $\Sigma^{2}$ in the $L^{1}$-norm, which is the boundary of an octahedron. The "top" and "bottom" facets are shaded.

$F_{t o p}$, the "top" facet, and call the antipodal facet the "bottom" facet $F_{b o t}$. Let $F_{m i d}$ denote the complement of $F_{\text {top }} \cup F_{b o t}$ in $\Sigma^{d}$, the "middle" band of the $d$-sphere. The strategy of our proof will be to assume for the sake of contradiction that a KKM cover of $\Delta^{d}$ has no common intersection point. Then we extend these sets to construct a closed cover of $\Sigma^{d}$ whose sets contain no pair of antipodes, thereby contradicting the LSB theorem.

Proof. Part (I). Construction. We first consider the case where a given KKM cover $C_{1}, \ldots, C_{d+1}$ of $\Delta^{d}$ is non-degenerate, i.e., for each $x$ in $\Delta^{d}$ and set $C_{i}, x$ is in $C_{i}$ only if $x_{i}>0$. In the alternate characterization of the KKM cover, this means that each face is only covered by the sets that correspond to the vertices spanning that face. For example, the figure at left in Figure 3 is degenerate because the white set covers a point on the bottom edge of the triangle.

For the sake of contradiction, assume that there is no point common to all the sets $C_{1}, \ldots, C_{d+1}$. For each $i$, let $-C_{i}$ be the set in $F_{b o t}$ antipodal to $C_{i}$. Let $B_{i}$ be the complement of $-C_{i}$ in $F_{b o t}$. By assumption every point of $F_{\text {top }}$ is excluded from at least one $C_{i}$. Hence the complementary sets $B_{i}$ form an open cover of $F_{b o t}$ (in the relative topology). Moreover, the sets $B_{i}$ satisfy a certain kind of non-degeneracy that follows from the non-degeneracy of the $C_{i}$ 's: for $x$ in $F_{b o t}, x_{i}=0$ implies that $x$ is covered by $B_{i}$. By normality, the sets $B_{i}$ can be shrunk to obtain closed subsets $E_{i}$ of $B_{i}$ that still cover $F_{b o t}$ and satisfy the same non-degeneracy.

Now that $F_{b o t}$ has been covered, we construct a cover of $F_{t o p} \cup F_{\text {mid }}$. For $x=\left(x_{i}\right)$ in $\Sigma^{d}$, let $\operatorname{pos}(x):=\sum_{x_{i}>0} x_{i}$. Note that $\operatorname{pos}(x)=0$ on $F_{b o t}$ but $\operatorname{pos}(x)>0$ on $F_{\text {top }}$ and $F_{\text {mid }}$. Define a function $f=\left(f_{i}\right)$ on $F_{\text {top }} \cup F_{\text {mid }}$ by:

$$
f_{i}(x)=\frac{x_{i}+\left|x_{i}\right|}{2 \operatorname{pos}(x)} \quad \text { if } \operatorname{pos}(x)>0 .
$$

Note that $f$ is a continuous function taking $F_{\text {top }} \cup F_{\text {mid }}$ to $F_{\text {top }}$, and it fixes $F_{\text {top }}$.

Then $D_{i}:=f^{-1}\left(C_{i}\right)$ is a closed subset of $F_{t o p} \cup F_{m i d}$ in the relative topology. We may think of the set $D_{i}$ as extending the set $C_{i}$ on $F_{\text {top }}$ to cover $F_{\text {mid }}$. In fact, $D_{i}$ extends the boundary of $C_{i}$ in a linear fashion across $F_{\text {mid }}$. See Figure 2 We record some observations about the sets $D_{i}$ :

Observation 1. Since the $C_{i}$ 's cover $F_{\text {top }}$, the $D_{i}$ 's cover $F_{\text {top }} \cup F_{\text {mid }}$. 
Observation 2. Since $f$ fixes $F_{\text {top }}$, each $D_{i}$ restricted to $F_{\text {top }}$ is just $C_{i}$.

Observation 3. If $x$ is in $D_{i}$, then $x_{i}>0$.

The first two observations are apparent from the definition of $f$, and the last observation follows by noting that if $x$ is in $D_{i}$, then $f(x)$ is in $C_{i}$ and the non-degeneracy of $C_{i}$ implies that $f_{i}(x)>0$. But this can only occur if $x_{i}>0$.

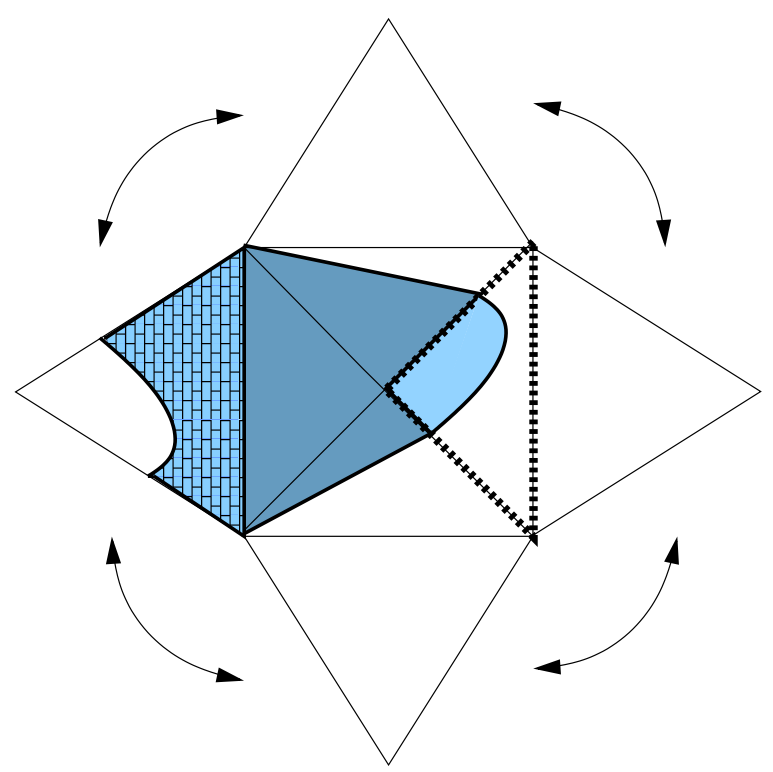

Figure 2. The octahedral 2-sphere $\Sigma^{2}$ unfolded, with shaded set $A_{i}$ derived from a set $C_{i}$ in the KKM cover. The set $A_{i}$ consists of three regions: light-shaded, dark-shaded, and bricked. The light-shaded region is $C_{i}$; it sits in $F_{\text {top }}$ (the triangle with dashed outline). The set $D_{i}$ extends $C_{i}$ and includes both the light-shaded and dark-shaded regions of $A_{i}$. The bricked region is $E_{i}$; it sits in the facet antipodal to $F_{\text {top }}$. Note its relation with $C_{i}$.

Now let $A_{i}=D_{i} \cup E_{i}$. We shall verify that the $A_{i}$ 's cover $\Sigma^{d}$ and are closed sets, yet no $A_{i}$ contains a pair of antipodes. This verification will contradict the LSB theorem, forcing us to reject our initial assumption that the KKM cover had no common intersection point.

Part (II). Verification. Clearly the $A_{i}$ 's cover $\Sigma^{d}$, by Observation 1 and the fact that the $E_{i}$ 's cover $F_{b o t}$.

To show $A_{i}$ is closed, note that $E_{i}$ is a closed subset of $\Sigma^{d}$ and $D_{i}$ is closed in $F_{\text {top }} \cup F_{\text {mid }}$ (but not necessarily $\Sigma^{d}$ ). Thus it suffices to show that any limit points of $D_{i}$ in $F_{b o t}$ must lie in $E_{i}$. Observation 3 implies that a limit point $x$ of $D_{i}$ must satisfy $x_{i} \geq 0$, but since points in $F_{b o t}$ have no positive coordinates, a limit point of $D_{i}$ in $F_{b o t}$ must satisfy $x_{i}=0$. By the non-degeneracy of $E_{i}, x$ must be in $E_{i}$.

To show that $A_{i}$ contains no pair of antipodes, we note that $E_{i}$ cannot contain a pair of antipodes and by Observation 3 neither can $D_{i}$, because for any $x$ in $D_{i}, x_{i}$ and $-x_{i}$ cannot both be positive. 


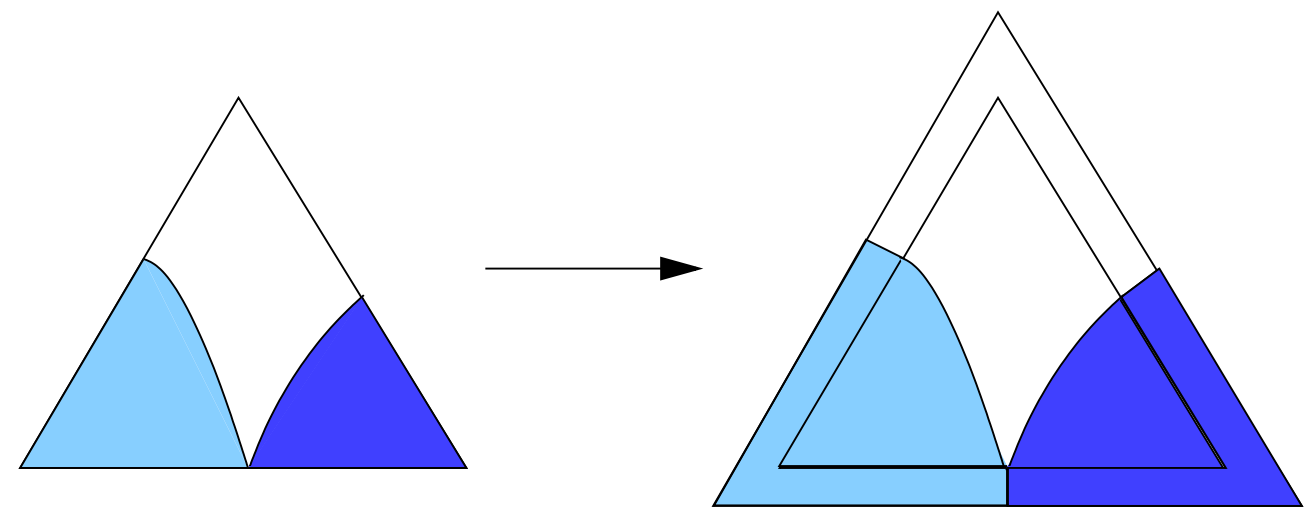

FiguRE 3. In these diagrams, the sets are closed and contain their boundaries. At left, the KKM cover is degenerate because the white (non-shaded set) covers a point on the bottom edge. At right, the same KKM cover has been "thickened" to form a non-degenerate KKM cover.

So all that remains is to check that there is no pair $x$ in $D_{i}$ and $-x$ in $E_{i}$. But this can only occur if $x$ is in $F_{t o p}$. By construction $C_{i}$ cannot have antipodes in $E_{i}$, so Observation 2 shows that $D_{i}$ has no antipodes in $E_{i}$. Hence the $A_{i}$ form a cover of $\Sigma^{d}$ by $d+1$ closed sets, yet no $A_{i}$ contains a pair of antipodes. This contradicts the LSB theorem.

Part (III). Degenerate KKM covers. Finally, we consider the case where the KKM cover is degenerate. We claim that a degenerate cover of $\Delta^{d}$ can be made non-degenerate by "thickening" up the boundary and extending the cover in a way that introduces no new common intersection point. Let $S$ be the subset of $\Delta^{d} \times[0,1]$ consisting of the points in $\Delta^{d} \times\{0\}$ and $\partial \Delta^{d} \times[0,1]$. (Here $\partial \Delta^{d}$ denotes the boundary of $\Delta^{d}$.) Thus $S$ is homeomorphic to $\Delta^{d}$; in fact, it is $\Delta^{d}$ with its boundary "thickened" up. Given a KKM cover of $\Delta^{d}$ by $C_{1}, C_{2}, \ldots C_{d+1}$, we construct a KKM cover $C_{1}^{\prime}, C_{2}^{\prime}, \ldots, C_{d+1}^{\prime}$ of $S$ that is non-degenerate. First, for $(x, 0)$ in $\Delta^{d} \times\{0\}$, put $(x, 0)$ in $C_{i}^{\prime}$ if $x$ is in $C_{i}$. Then, for $(x, t)$ in $\partial \Delta^{d} \times[0,1]$ where $t>0$, put $(x, t)$ in $C_{i}^{\prime}$ if $x$ is in $C_{i}$ and $x_{i}>0$. One may check that the $C_{i}^{\prime}$ 's are closed and by construction there are no points of $\cap C_{i}^{\prime}$ in the portions of $S$ where $t>0$. See Figure 3 This "thickened", non-degenerate cover can then be used as in the first part of this proof.

We remark that although our proof of the KKM lemma appears non-constructive, the asserted KKM intersection is hiding in our construction in the following way. When we assume (falsely) that the asserted KKM intersection does not exist, we are (wrongly) led to conclude that the $B_{i}$ 's cover the bottom facet of $\Sigma^{d}$. In actuality, these open sets do not cover the bottom facet; the set of points that are exposed are precisely the points whose antipodes comprise the asserted KKM intersection in the top facet.

\section{REFERENCES}

[1] I. Bárány, A short proof of Kneser's conjecture, J. Combin. Theory Ser.A 25 (1978) 325-326.

[2] K. C. Border, Fixed point theorems with applications to economics and game theory, Cambridge Univ. Press, Cambridge, 1985. 
[3] K. Borsuk, Drei Sätze über die n-dimensionale euklidische Sphäre, Fund. Math. 20 (1933) 177-190.

[4] J. Greene, A new short proof of Kneser's conjecture, Amer. Math. Monthly 109 (2002) 918-920.

[5] B. Knaster, C. Kuratowski, and S. Mazurkiewicz, Ein Beweis des Fixpunktsatzes für $n$-dimensionale Simplexe, Fund. Math. 14 (1929) 132-137.

[6] L. Lusternik and L. Schnirelman, Topological Methods in Variational Calculus, Issledowatelskii Institut Matematiki i Mechaniki pri O.M.G.U., Moscow, 1930 [Russian].

[7] F. E. Su, Borsuk-Ulam implies Brouwer: a direct construction, Amer. Math. Monthly 104 (1997) 855-859.

Department of Mathematics, Harvey Mudd College, Claremont, CA 91711

E-mail address: gspencer@hmc.edu

Department of Mathematics, Harvey Mudd College, Claremont, CA 91711

E-mail address: su@math.hmc.edu 